\title{
Simulation of a Contact Pseudo-Environment in Calculating Thermal Resistance
}

\author{
Alexander Denisenko, ${ }^{1,}$, Roman Grishin ${ }^{1}$, and Liubov Podkruglyak ${ }^{1}$ \\ ${ }^{1}$ Samara State Technical University, Samara, Russian Federation
}

\begin{abstract}
The use of the temperature criterion in the design of metalcutting machines, determined on the basis of models that take into account the contact thermal resistances, is an objective necessity. These models should take into account to the maximum extent the actual conditions of contact of parts in the design under consideration, determined by the deviations of the mating surfaces from the ideal shape.The article presents the results of numerical modeling based on the finite element method of the formation of the contact thermal resistance and the evaluation of the influence of the parameters of the intermediate layer (pseudo-environment) that occurs in the contact zone of surfaces with macro-deviations on the passage of the heat flow. The obtained results allowed us to identify the most significant of the considered parameters. It is established that when modeling a pseudo-environment, it is necessary to take into account the coefficient of its thermal conductivity, the size, location and integrity of the actual contact zone.
\end{abstract}

\section{Introduction}

Analysis of the metalworking error balance shows that the thermal error is $40 \ldots 70 \%$ of the total manufacturing error. In this regard, the constantly increasing requirements for the accuracy of processing on metal-cutting machines put thermal processes among the main factors that determine the quality of products.

Modeling the flow of heat flows in the design of a metal-cutting mill is difficult due to the large number of heat sources (electric motors, rolling bearings, cutting zone, gears and belt drives, hydraulic system, etc.) and the large number of parts included in their design. The latter circumstance makes it necessary to take into account in the thermal model the passage of heat flows from sources through solid parts and their contacts with each other [1, 2].

The passage of heat flow through the actual contacts of the parts is very different from its movement through a solid material and through an ideal joint, when the heat flow meets almost no significant resistance in its path. The reason is that in real contacts, due to the presence of macro-deviations, undulations and roughness, which depend on the structure and nature of the processing, a continuous contact is broken along the joint. This leads to the appearance of contact thermal resistance (CTR), which must be taken into account in

\footnotetext{
* Corresponding author: sammortor@yandex.ru
} 
the thermal models used. Thus, the need to reasonably manage heat flows in particularly precise nodes of metal-cutting machines due to design and technological measures make the task of modeling the CTR very relevant.

\section{Using a pseudo-environment to determine the CTR}

As it was shown in [1], the introduction to the thermal model in the modeling of CTR connections makes it possible, using the electrothermal analogy, to use large-block finite elements and significantly simplify the preparation of the model, which is especially important at the stage of designing and analyzing various options for the design of the node. Thus, the design component can be taken into account in the analysis according to the temperature criterion, which is primarily related to the shape and size of fixed joints. In addition, such a model also makes it possible to assess the impact of the quality requirements of the contacting surfaces, determined by the deviations of the mating surfaces from the ideal shape, which the designer often assigns intuitively or on the basis of established recommendations, which, with rare exceptions, take into account the temperature criterion.

Previous studies by a number of authors [3...10] have shown that the contact conditions have a very significant effect on the passage of heat flow in machine tool structures.

When studying complex processes, as a rule, you have to deal with the action of a large number of factors. For example, in [9], the dependences of the thermal resistance of the contact are given, taking into account the mean square deviation of the profile; the hardness (microhardness) of the surface; the average tangent of the angle of inclination of the roughness; the maximum radius of rounding of the tips of the protrusions; a parameter that characterizes the degree of mechanical loading of the contact irregularities. The degree of influence and weight of the active factors, as a rule, is different. Therefore, when developing a mathematical model for the formation of CTR, it is necessary to conduct preliminary studies that allow us to identify the significant factors that affect the contact thermal resistance. Without this procedure, generally accepted methods (for example, regression analysis) with a large number of factors become unacceptable, since they require a large number of experiments.

To this end, a number of univariate experiments were conducted.

The modern theory of surface contact is based on the position that the presence of roughness, undulation and macro-deviations leads to the discreteness of their interaction. In addition, when contacting rough bodies, it should be taken into account that, despite the different heights, all the protrusions are elastically deformed, but to different degrees. The result is a layer (pseudo-environment) consisting of the actual contact areas and cavities filled with air or oil.

\section{Development of a finite element model for contacting two plates}

To assess the influence of the characteristics of the pseudo-environment at the contact of two solid bodies on the conditions for the formation of the CTR, a flat finite element model of the contact of two square plates $(0.02 \mathrm{~m} \mathrm{x} 0.02 \mathrm{~m})$ with a thickness of $1 \mathrm{~mm}$ was developed (Fig. 1). The material of the plates is steel (thermal conductivity coefficient $\lambda=50 \mathrm{~W} /(\mathrm{m} \cdot \mathrm{K}))$. The power of each of the five heat sources is $20 \mathrm{~W} / \mathrm{m}$. The boundary conditions for the edges of the model were assumed as follows: the convection 
$F_{n}=\alpha\left(T-T_{0}\right)$ along the side edges and along the upper edge was zero $\left(\alpha=0\right.$ and $\left.T_{0}=0\right)$, and along the lower edge $\alpha=50 \mathrm{~W} / \mathrm{K} \cdot \mathrm{m} 2$ and $T_{0}=293 \mathrm{~K}$.

Numerical experiments with the developed finite element model were carried out using the ELCUT package [11...15].

The thermal resistance of the pseudo-environment $R, \mathrm{~K} \cdot \mathrm{m}^{2} / \mathrm{W}$, was determined by the formula $R=\Delta T / q$, where $q$ is the heat flux, $\mathrm{W} / \mathrm{m}^{2} ; \Delta T$ is the temperature drop.

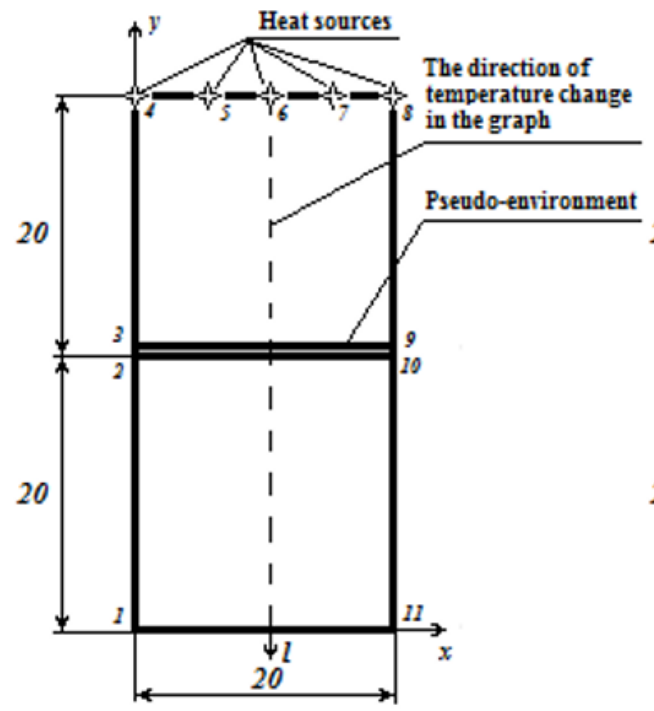

a)

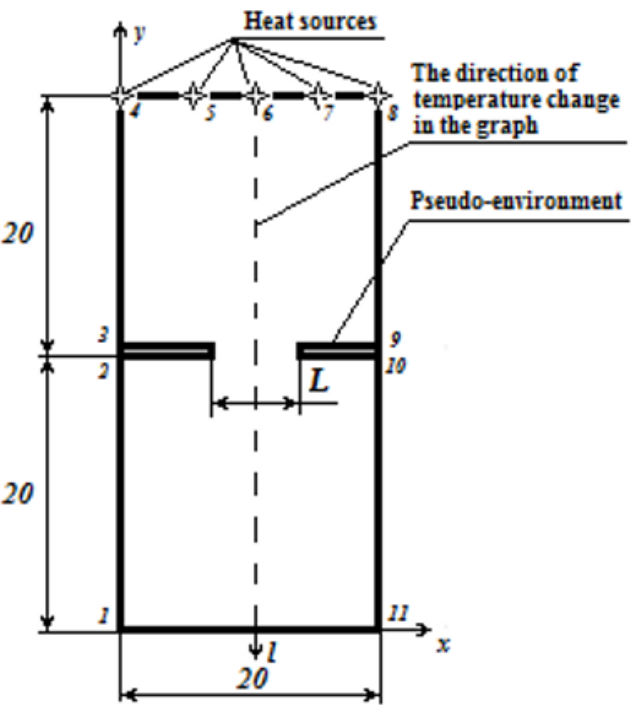

b)

Fig. 1. Flat model of contacting two plates: a) a pseudo-environment with a thermal conductivity coefficient different from the base material; b) a pseudo-environment that has actual contact on a part of the surface.

\section{Numerical simulation results}

\subsection{Contact details that do not have macro deviations}

For plates that do not have macro-deviations in the contact zone, the change in thermal conductivity in the contact zone will be determined only by micro-deviations, which can be assumed to be the same throughout the entire connection zone. Thus, in this case, there is a continuous pseudo-environment between the parts, which has a thermal conductivity coefficient and a thickness that depends on the roughness of the contacting surfaces.

Since the thermal conductivity coefficient of the pseudo-environment is formed as a result of direct contact of micro-roughnesses with the thermal conductivity coefficient of the base material, and the presence of cavities filled with oil or air, it can vary from $\lambda$ $=0.025 \mathrm{~W} /(\mathrm{m} \cdot \mathrm{K})$ (air) up to $\lambda=50.0 \mathrm{~W} /(\mathrm{m} \cdot \mathrm{K})$ (steel). The resulting dependence of $\Delta T$ on the thermal conductivity coefficient is shown in Fig. 2.

To estimate the effect of the thickness of the pseudo-environment $\delta$ on the values of $\Delta T$, numerical experiments were performed for the pseudo-layer with $\lambda=5 \mathrm{~W} /(\mathrm{m} \cdot \mathrm{K})$ and $\lambda=0.025 \mathrm{~W} /(\mathrm{m} \cdot \mathrm{K})$.

The simulation results presented in Table 1 indicate the linear nature of the dependence $\Delta T=f(\delta)$. 


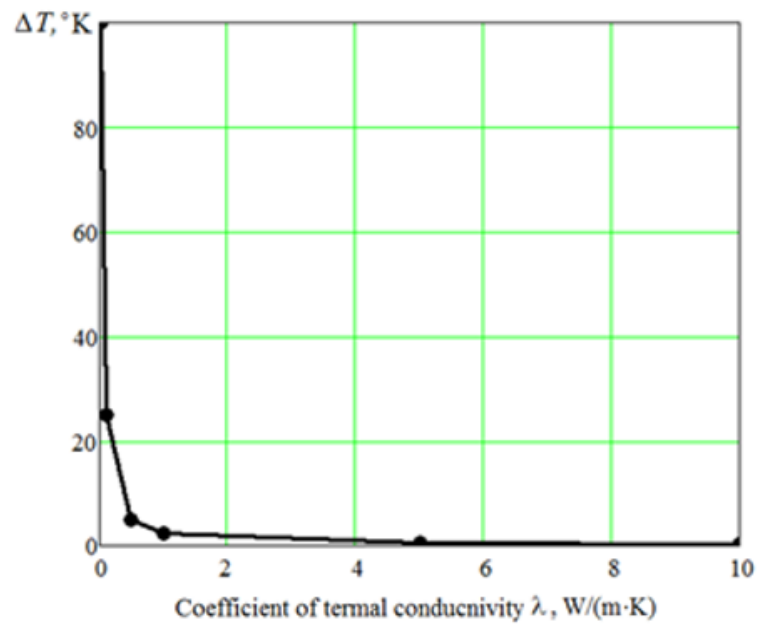

Fig. 2. Change of $\Delta T$ depending on the thermal conductivity coefficient of the pseudo-environment.

Table 1. Dependence of the temperature drop in the contact on the thickness and quality of the pseudo-environment.

\begin{tabular}{|c|c|c|}
\hline \multirow{2}{*}{$\delta, \mathrm{mm}$} & \multicolumn{2}{|c|}{$\Delta T$} \\
\cline { 2 - 3 } & $\lambda=5 \mathrm{~W} /(\mathrm{m} \cdot \mathrm{K})$ & $\lambda=0.025 \mathrm{~W} /(\mathrm{m} \cdot \mathrm{K})$ \\
\hline 0.5 & 0.45 & 100 \\
\hline 0.4 & 0.35 & 80 \\
\hline 0.3 & 0.27 & 60 \\
\hline 0.2 & 0.18 & 40 \\
\hline 0.1 & 0.09 & 20 \\
\hline 0.07 & 0.06 & 14 \\
\hline
\end{tabular}

\subsection{Contact of parts with macro-deviations in the form of an axisymmetric bulge}

With an increase in the deviation from the flatness, the actual contact zone (ACZ), the thermal conductivity of which is equal to the thermal conductivity of the material of the contacting bodies, decreases.

To estimate the passage of heat flow through a pseudo-environment that has actual contact on a part of the surface, a flat model with a thickness of $1 \mathrm{~mm}$ was used, shown in Fig. 1, b. The length of the ACZ was assumed to be 1, 2, 3, and $4 \mathrm{~mm}$. The coefficient of thermal conductivity in the ACZ was taken as for the main material (the material of the parts), and for the pseudo-environment two options were considered: $\lambda=0.025 \mathrm{~W} /(\mathrm{m} \cdot \mathrm{K})$ and $\lambda=5 \mathrm{~W} /(\mathrm{m} \cdot \mathrm{K})$.

The results presented in the form of dependencies $\Delta T=f(L)$ (Fig. 3) show a qualitatively identical picture of the dependencies for both values of $\lambda$, and quantitativelysignificantly dependent on the thermal conductivity coefficient of the pseudo-environment outside the ACZ. So, for example, for a pseudo-environment with $\lambda=0.025 \mathrm{~W} /(\mathrm{m} \cdot \mathrm{K})$, the change in the length of the ACZ from 1 to $4 \mathrm{~mm}$ led to a decrease in $\Delta T$ approximately twice, and for a pseudo-environment with $\lambda=5 \mathrm{~W} /(\mathrm{m} \cdot \mathrm{K})$ - only by $22 \%$. 


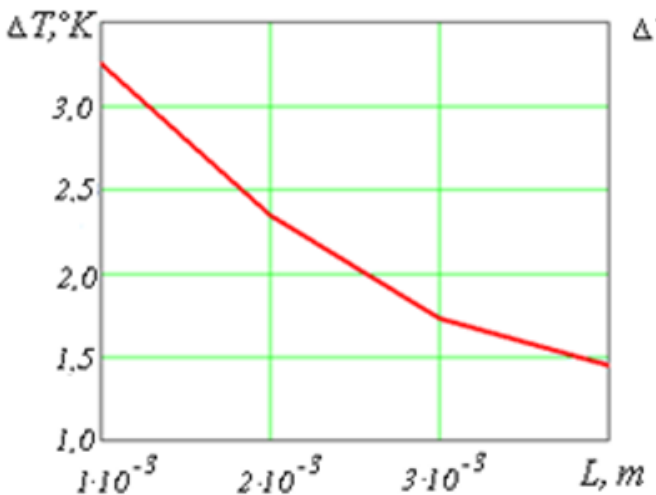

a)

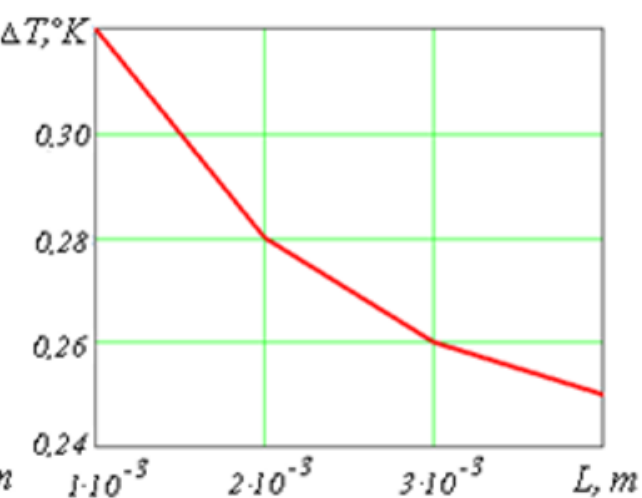

b)

Fig. 3. Dependence on the length of the ACZ for the pseudo-environment with $\lambda=0.025 \mathrm{~W} /(\mathrm{m} \cdot \mathrm{K})$ (a) and $\lambda=5 \mathrm{~W} /(\mathrm{m} \cdot \mathrm{K})(\mathrm{b})$.

\subsection{Contact of parts with macro-deviations in the form of a non-axisymmetric bulge}

To estimate the passage of heat flow through a contact with a non-axisymmetric SFK arrangement, a model with $L=2 \mathrm{~mm}$ and a pseudo-environment filled with air was considered for three variants of the ACZ arrangement (Fig.4).

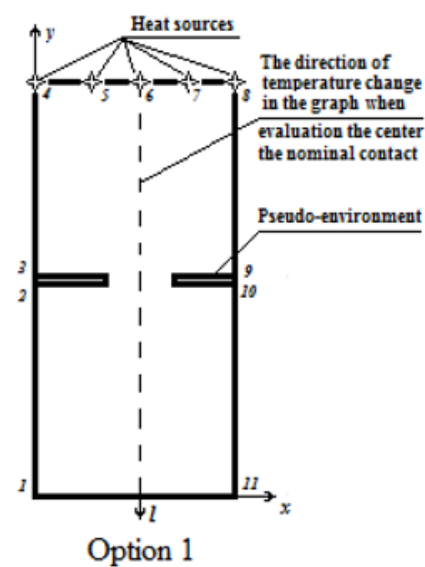

a)

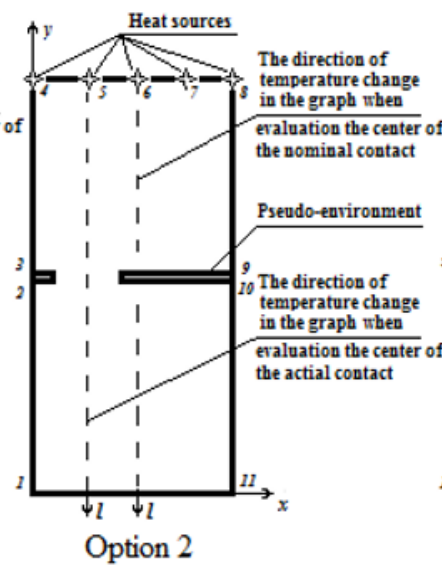

b)

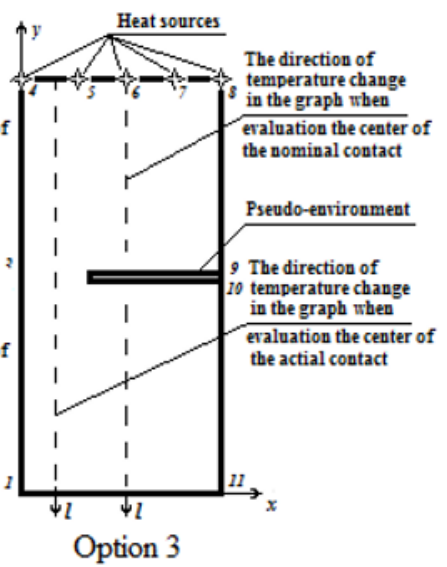

c)

Fig. 4. Flat model of contact between two plates with axisymmetric (a) and non-axisymmetric (b and c) ACZ arrangement..

The simulation results are shown in Fig. 5 and in Table 2.

Thus, the value of $\Delta T$ depends on the position of the ACZ in the connection. In addition, the qualitative picture of the change in $\Delta T$ significantly depends on the location of the line along which this assessment is made: the assessment at the center of the actual contact is accompanied by a sudden change in temperature when the heat flow passes through the pseudo-environment, in contrast to the assessment at the center of the nominal contact, where this difference is more smoothly (Fig.5). 


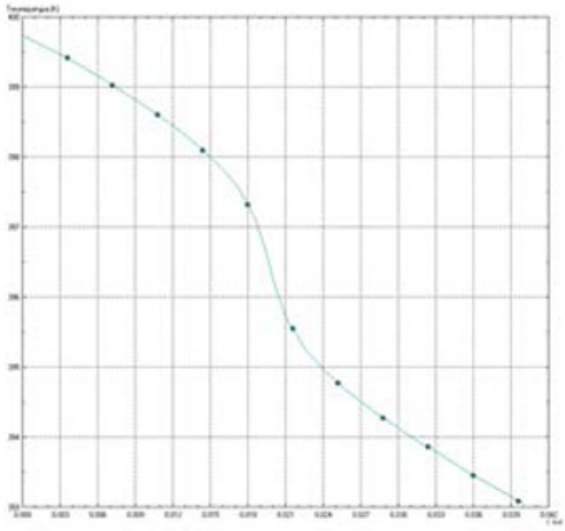

a)

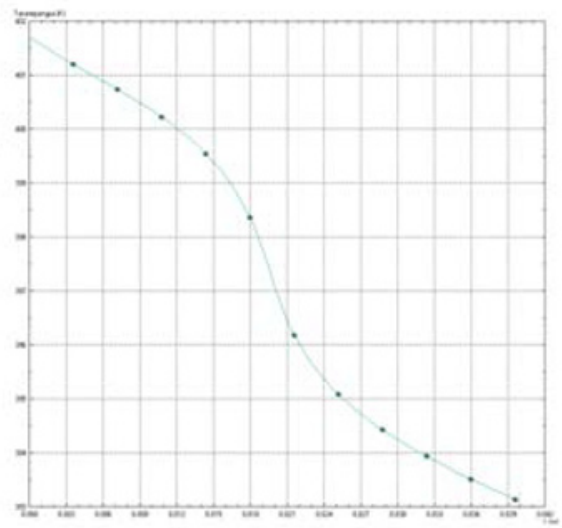

c)

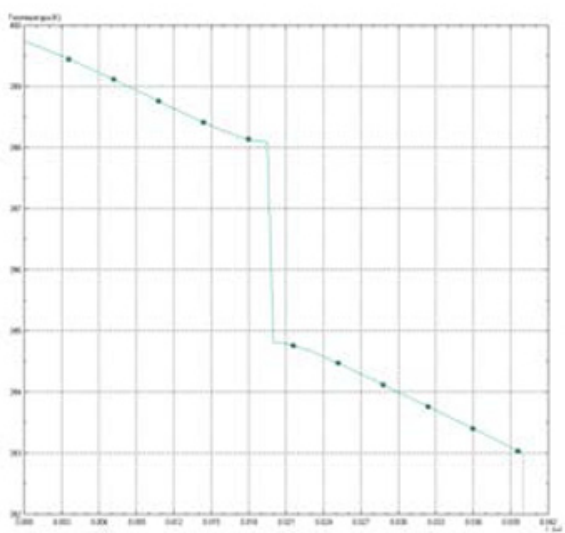

b)

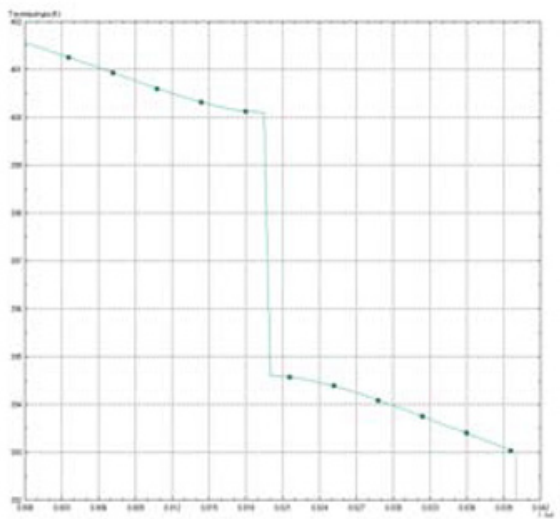

d)

Fig. 5. Graphs of temperature changes for variants 2 (a and b) and 3 (c and d) with the axis $l$ located at the center of the ACZ ( $a$ and $b$ ) and at the center of the junction (b and d), obtained from the results of modeling in the ELCUT environment.

Table 2. Dependence of the temperature drop in the contact on the location of the ACZ.

\begin{tabular}{|c|c|c|c|c|c|}
\hline $\begin{array}{c}\text { Option for the } \\
\text { location of } \\
\text { the ACZ }\end{array}$ & Option 1 & \multicolumn{2}{|c|}{ Option 2 } & \multicolumn{2}{c|}{ Option 3 } \\
\hline$\Delta T,{ }^{\circ} \mathrm{K}$ & 2.35 & $\begin{array}{c}\text { Assessment at } \\
\text { the center of } \\
\text { the actual } \\
\text { contact }\end{array}$ & $\begin{array}{c}\text { Assessment at } \\
\text { the center of } \\
\text { the nominal } \\
\text { contact }\end{array}$ & $\begin{array}{c}\text { Assessment at } \\
\text { the center of } \\
\text { the actual } \\
\text { contact }\end{array}$ & $\begin{array}{c}\text { Assessment at } \\
\text { the center of } \\
\text { the nominal } \\
\text { contact }\end{array}$ \\
\hline
\end{tabular}

\subsection{Connecting parts that have a non-continuous $A C Z$}

To check the effect of the type of ACZ (distributed or concentrated) on $\Delta T$, we compare two cases with a pseudo-environment filled with air (Fig. 6), provided that the total length of the actual contact remains constant:

1) centrally located ACZ with a length of $L=0.003$ m (concentrated ACZ);

2) three ACZ, the total length of which is $L=0.001 \cdot 3=0.003 \mathrm{~m}$ (distributed ACZ). 


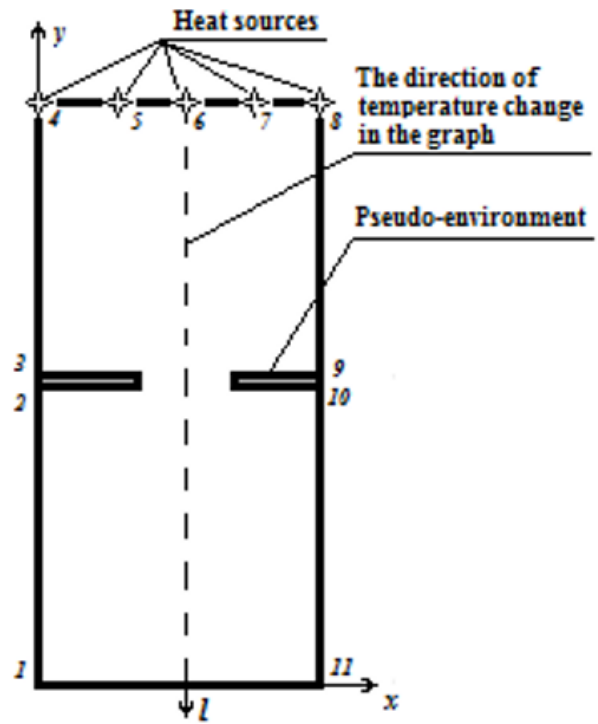

a)

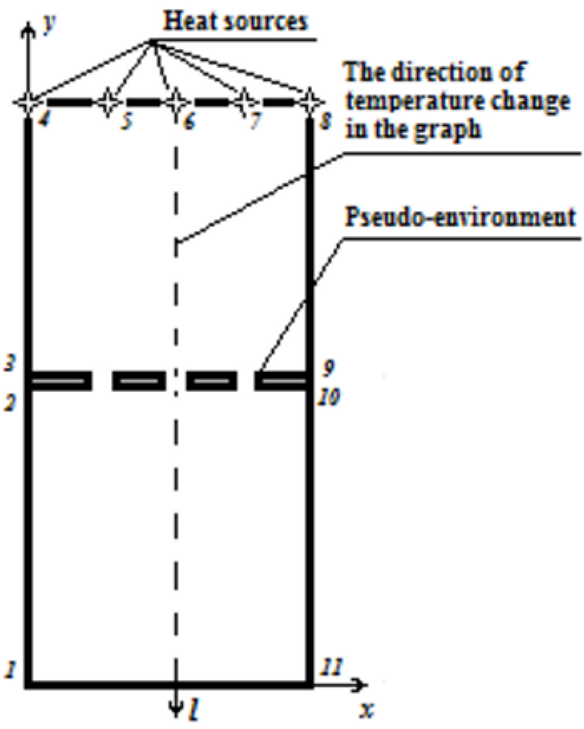

b)

Fig. 6. A flat model of the contact of two plates with a concentrated (a) and distributed (b) ACZ.

The simulation results (Fig. 7) showed that while maintaining the total size of the ACZ, the number of ACZ significantly affects $\Delta T$ : when they increase, the value of $\Delta T$ decreases.

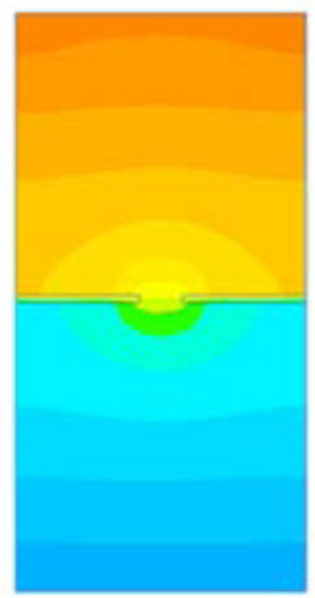

a)
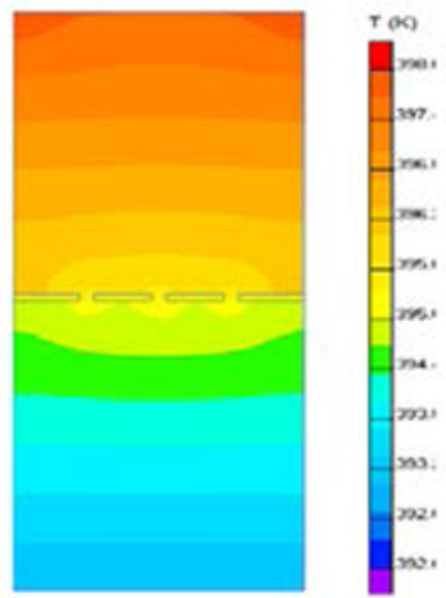

b)

Fig. 7. Results of numerical simulation in the ELCUT environment with a concentrated ACZ length of $3 \mathrm{~mm}$ (a) and a distributed ACZ total length of $3 \mathrm{~mm}$ (b).

\section{Conclusions}

From the conducted numerical experiments, it can be concluded that with a decrease in the thickness of the pseudo-medium and an increase in the thermal conductivity coefficient, i.e., an improvement in the quality of the contact, the temperature drop in the contact $\Delta T$ 
decreases, and the contact thermal resistance decreases accordingly. Moreover, the CTR depends linearly on the thickness of the pseudo-layer, and the influence of the thermal conductivity coefficient of the pseudo-environment has a pronounced nonlinear character.

It is found that with a decrease in the deviation from the flatness, when the length of the $\mathrm{ACZ}$ increases, the temperature difference in the contact $\Delta T$ decreases. The decrease in $\Delta T$ with an increase in the length of the ACZ significantly depends on the thermal conductivity coefficient of the pseudo-environment.

A comparison of the results of the influence of macro-deviations on the CTR, when a concentrated ACZ is created, and undulation, when there is a distributed ACZ of the same total area, showed a more significant role of macro-deviations.

\section{References}

1. A.F. Denisenko, L.Y. Podkruglyak, Bulletin of the Samara Scientific Center of the Russian Academy of Sciences, 22(3), pp.49-55 (2020)

2. A.F. Denisenko, L.Y. Podkruglyak, IOP Conf. Series: Materials Science and Engineering 971022020 (2020). doi:10.1088/1757-899X/971/2/022020

3. S.Yu. Mesnyankin, A.G. Vikulov, D.G. Vikulov, Advances in physical sciences, 179(9), pp. 945-970 (2009)

4. S. Kamenev, IOP Conf. Ser.: Mater. Sci. Eng. 327022055 (2018)

5. A. Lipov, G. Bolshakov, V. Panchurin, Models, systems, networks in economics, technike, nature and society, 4 (12), pp. 124-128 (2014)

6. V.R. Prabhu, R.M. Sathiya, Arabian Journal for Science and Engineering. https://doi.org/10.1007/s13369-019-03732-x (2019)

7. Li Yang et al, International Journal of Machine Tools \& Manufacture, 95, pp. 20-38 (2015)

8. Li Wu, Qingchang Tan Study of a Spindle-Bearing System Entropy 18, 271, pp.1-25

9. V. Izmailov, S. Chaplygin, Internet journal "SCIENCE", 8(2) (2016) http://naukovedenie.ru/PDF/26TVN216.pdf (free access). DOI: 10.15862 / 26TVN216

10. A. Denisenko, N. Nazarov, Assembly in mechanical engineering, instrument making, 7, pp. 325-329 (2017)

11. I.A. Salova, V.V. Khrushchev, Simulation in ELCUT. - Saint Petersburg: Saint Petersburg State University of Aerospace Instrumentation (2007)

12. ELCUT. Simulation of electromagnetic, thermal and elastic fields by the finite element method. Version 6.0. User's manual. LLC "Tor", Saint Petersburg (2013)

13. A.N. Semernin, A.A. Urvanov, Energy systems, 1, pp. 289-291(2016)

14. A.S. Tatevosyan et, International Journal of Applied and Basic Research, 11-1, pp. 19$24(2015)$

15. E.G. Andreeva, A.A. Tatevosyan, I.A. Semina, Dynamics of systems, mechanisms and machines 1, pp. 111-114 (2009) 